\title{
Efecto de expansión rápida del maxilar en el tratamiento de trastornos respiratorios del sueño en niños, revisión bibliográfica
}

Effect of rapid expansion of the maxilla in the treatment of sleep breathing disorders in children, bibliographic review

Efeito da expansão rápida da maxila no tratamento dos distúrbios respiratórios do sono em crianças, revisão de literatura

\author{
Antonieta Pérez-Flores ${ }^{1}$ \\ Ricardo Alarcón ${ }^{2}$ \\ Lorena Bravo ${ }^{3}$ \\ Claudia Fierro-Monti ${ }^{4}$ \\ Romina Novoa ${ }^{5}$
}

Recibido: 3 de marzo de 2020

Aprobado: 12 de abril de 2021

Publicado: 4 de junio de 2021

Cómo citar este artículo:

Perez-Flores A, Alarcón R, Bravo L, Fierro-Monti C, Novoa R. Efecto de Expansión rápida del maxilar en el tratamiento de trastornos respiratorios del sueño en niños, revisión bibliográfica. Revista Nacional de Odontología. (2021); 17(1), 1-11. doi: https://doi.org/10.16925/2357-4607.2021.01.09

\footnotetext{
Revisión de literatura. https://doi.org/10.16925/2357-4607.2021.01.09

1 Universidad de Concepción, Chile. ORCID: https://orcid.org/0000-0002-2481-7088

Correo electrónico: mperezf@udec.cl

2 Universidad de Concepción, Chile.

ORCID: https://orcid.org/0000-0002-8260-7441

3 Universidad de Concepción, Chile. ORCID: https://orcid.org/0000-0002-7692-7684

4 Universidad de Concepción, Chile. ORCID: https://orcid.org/0000-0002-0308-6044

5 Hospital Clínico Guillermo Grant Benavente ORCID: https://orcid.org/0000-0002-2481-7088
} 
2 Efecto de Expansión rápida del maxilar en el tratamiento de trastornos respiratorios del sueño en niños, revisión bibliográfica

\title{
Resumen
}

El sueño y la respiración son intrínsecamente funciones relacionadas. El espectro de los Trastornos Respiratorios del Sueño (TRS) incluye: el Síndrome de Apnea Obstructiva del Sueño (SAOS), Síndrome de Resistencia de Vía Aérea Superior (SRVAS) y Ronquido Primario (RP). Si bien el ronquido es una manifestación muy frecuente, su presencia evidencia algún grado de obstrucción parcial de la vía aérea superior. El tratamiento quirúrgico, adenotonsilectomía, ha sido recomendado como primera línea por guías clínicas extranjeras. La susceptibilidad anatómica para desarrollar apnea del sueño se determina por la relación entre las dimensiones fijas del esqueleto craneofacial, volumen y distribución de las estructuras de los tejidos blandos y el tejido adiposo que residen en el compartimiento esquelético. La expansión rápida del maxilar se utiliza actualmente en niños que presentan constricción maxilar y como tratamiento alternativo de TRS en niños. Se presenta la evidencia bibliográfica de los últimos 15 años para tratamiento ortopédico de expansión maxilar como tratamiento coadyuvante de TRS en la infancia.

Palabras clave: expansión rápida del maxilar, trastornos respiratorios del sueño en niños.

\begin{abstract}
Sleep and breathing are intrinsically related functions. The spectrum of Sleep Respiratory Disorders (SRD) includes: Obstructive Sleep Apnea Syndrome (OSAS), Upper Airway Resistance Syndrome (UARS) and Primary Snoring (PS). Although snoring is a very frequent manifestation, its presence shows some degree of partial obstruction of the upper airway. Surgical treatment, adenotonsilectomy, has been recommended as a first line by foreign clinical guidelines. The anatomical susceptibility to develop sleep apnea is determined by the relationship between the fixed dimensions of the craniofacial skeleton, volume and distribution of soft tissue structures and adipose tissue that reside in the skeletal compartment. The rapid expansion of the maxilla is currently used in children with maxillary constriction and as an alternative treatment for SRD in children. The bibliographic evidence of the last 15 years is presented for orthopedic treatment of maxillary expansion as an adjunctive treatment of SRD in childhood.
\end{abstract}

Key words: rapid maxillar expansion, sleep breathing disorders in children

\section{Resumo}

O sono e a respiração são funções intrinsecamente relacionadas. 0 espectro dos distúrbios respiratórios do sono (SRB) inclui: Síndrome da Apnéia Obstrutiva do Sono (SAOS), Síndrome de Resistência das Vias Aéreas Superiores (SRVAS) e Ronco Primário (RP). Embora o ronco seja uma manifestação muito comum, sua presença evidencia algum grau de obstrução parcial da via aérea superior. 0 tratamento cirúrgico, adenotonsilectomia, tem sido recomendado como primeira linha por diretrizes clínicas estrangeiras. A suscetibilidade anatômica ao desenvolvimento da apnéia do sono é determinada pela relação entre as dimensões fixas do esqueleto craniofacial, volume e distribuição das estruturas de tecidos moles e tecido adiposo que reside no compartimento esquelético. A expansão rápida da maxila é atualmente utilizada em crianças com constrição maxilar e como tratamento alternativo para TRS em crianças. São apresentadas as evidências bibliográficas dos últimos 15 anos para o tratamento ortopédico da expansão maxilar como tratamento adjuvante do TRS na infância.

Palavras-chave: expansão rápida da maxila, distúrbios respiratórios do sono em crianças. 


\section{Introducción}

El sueño es un estado fisiológico distinto a la vigilia, en el que se manifiestan cambios en la actividad cerebral, el tono muscular y la función autonómica (control cardíaco y respiratorio). La arquitectura del sueño, tiempo total de sueño y puesta en escena del sueño, difieren considerablemente desde la vida fetal hasta la edad adulta. El sueño y la respiración son funciones intrínsecamente relacionadas, y esta relación es fundamental para la comprensión de los trastornos respiratorios del sueño (TRS) en la infancia (1). El objetivo de este artículo fue realizar una revisión bibliográfica para el enfoque terapéutico ortopédico y la expansión rápida del maxilar en el tratamiento de los TRS en niños.

\section{Revisión y métodos}

Se realizó una revisión narrativa, con inclusión de artículos con análisis de revisiones sistemáticas, revisiones bibliográficas, reporte de casos clínicos, estudios longitudinales y de caso-control; y guías clínicas odontológicas chilenas sobre la siguiente pregunta de búsqueda: ¿cuál es el enfoque ortopédico en el tratamiento de trastornos respiratorios del sueño en niños?

\section{Proceso de selección}

La selección la realizaron dos revisores de forma independiente. Se seleccionaron los títulos y se eliminaron las publicaciones no pertinentes. Se utilizaron los filtros de cada base de datos seleccionando las opciones de "búsqueda por tiempo", "búsqueda de ensayos clínicos" y "búsqueda de artículos" principalmente en las bases PubMed y ScienceDirect. Los desacuerdos entre los dos revisores se resolvieron con una discusión adicional.

La búsqueda de la literatura se realizó mediante buscadores de literatura biomédica: PUBMED, SCIELO, Medline, combinando palabras claves: 'trastornos respiratorios del sueño en niños' y 'expansión rápida del maxilar'.

Se seleccionaron 32 artículos que cumplían con los siguientes criterios de inclusión artículos completos en español e inglés. Los artículos fueron seleccionados dentro de los 15 últimos años.

Como criterios de exclusión se eliminaron artículos repetidos, cartas al editor o comentarios y artículos que no tuviesen texto completo disponible. 
4 Efecto de Expansión rápida del maxilar en el tratamiento de trastornos respiratorios del sueño en niños, revisión bibliográfica

\section{Desarrollo}

\section{Trastornos respiratorios del sueño}

El espectro de los trastornos respiratorios del sueño (TRS) incluye el Síndrome de Apnea Obstructiva del Sueño (SAOS), el Síndrome de Resistencia de la Vía Aérea Superior (SRVAS) y el Ronquido Primario (RP). El RP por definición no se acompaña de apneas, hipopneas, desaturaciones ni microdespertares electroencefalográficos. Si bien el ronquido es una manifestación muy frecuente, no constituye un evento fisiológico, por el contrario, su presencia evidencia algún grado de obstrucción parcial de la vía aérea superior. Hasta hace apenas un par de décadas se pensaba que en aquellas ocasiones, en que el ronquido se presentaba en forma aislada, no generaba repercusión alguna en el estado de salud del paciente, constituyendo tan solo un problema social para su acompañante en la cama. Sin embargo, actualmente se describe que el SRVAS en pacientes roncadores que no presentan apneas ni hipoapneas, genera altas presiones negativas a nivel de vía aérea y esófago, lo que induce microdespertares con la consiguiente fragmentación del sueño, originando la hipersomnia diurna que aqueja a estos pacientes. En los últimos años se ha publicado una serie de investigaciones que sustentan un nuevo enfoque, según el cual el RP no sería tan "benigno" como se creía hasta hace algunos años, sino por el contrario, produciría un significativo deterioro de las funciones cognitivas y académicas de quienes lo presentan, generando además alteraciones conductuales e incluso desordenes metabólicos, especialmente en niños (1-3).

Por su parte, el SAOS se caracteriza por la oclusión intermitente, parcial o completa, de la vía aérea superior durante el sueño, produciendo alteración del intercambio gaseoso, frecuentes microdespertares y alteración de la arquitectura del sueño $(4,5)$. La presentación clínica del SAOS en niños difiere de la presentación clásica y de más fácil diagnóstico en adultos, por lo que requiere una agudeza en la sospecha clínica, observando cualquier síntoma nocturno, como el aumento del esfuerzo respiratorio, apneas o pausas respiratorias, sueño intranquilo, posturas anormales al dormir para mantener una vía aérea permeable, enuresis, bruxismo y sudoración nocturna. También se pueden observar síntomas diurnos que incluyen sequedad bucal, obstrucción nasal asociada a respiración bucal, somnolencia diurna, hiperactividad y problemas neurocognitivos. (6)

Muestras de gran tamaño reportaron tasas de prevalencia a nivel mundial de SAOS en niños de $1,2 \%$ a 5,7\%. $(3,6,7)$.

La ocurrencia del estrechamiento o cierre de la vía aérea superior durante el sueño se produce como resultado de anormalidades anatómicas y funcionales de 
la regulación de la vía aérea superior. La susceptibilidad anatómica para la apnea del sueño se determina por la relación entre las dimensiones fijas del esqueleto craneofacial, el volumen y distribución de las estructuras de los tejidos blandos y el tejido adiposo que residen en el compartimiento esquelético, es decir, mientras más comprometidas estén las estructuras anatómicas, más riesgo existe de tener un TRS y, así mismo, de aumentar sus consecuencias (8).

\section{Consecuencias de TRS}

Los TRS se han asociado a una serie de complicaciones en vigilia a largo plazo. Cabe destacar, que incluso los niños "roncadores primarios" presentarían una disrupción de la microarquitectura del sueño, pudiendo ocasionar consecuencias neurológicas. Existe evidencia que los TRS en niños mayores se asocian a un menor rendimiento escolar (2). Estos déficits cognitivos o neuropsicológicos se presentan también en asociación con síntomas, signos o diagnóstico de los TRS, es decir, un bajo rendimiento académico podría ser un signo de sospecha de TRS. Entre los déficits específicos medidos objetivamente se incluyen: aprendizaje, memoria y habilidades visuo-espaciales; idioma, fluidez verbal y habilidades fonológicas; formación de conceptos, pensamiento analítico, comprensión verbal y no verbal; rendimiento escolar y habilidades matemáticas (9-12). Así mismo, la mayoría de las investigaciones han demostrado que estos índices mejoran después del tratamiento de los TRS, en cuanto a la calidad de vida, hiperactividad e impulsividad (10).

Por otro lado, la presencia de TRS a largo plazo puede producir un compromiso cardiovascular de importancia. El desarrollo de hipertensión arterial crónica se ha asociado a TRS en la infancia (13). Así también, se ha descrito la aparición de hipertensión pulmonar y corpulmonale en pacientes roncadores no tratados (14). Estas complicaciones se producirían por los efectos de la hipoxemia intermitente sobre el sistema simpático y vasomotor. En pacientes con enfermedades neuromusculares de base, los TRS pueden producir estas repercusiones de forma aún más precoz (15).

Estas repercusiones adquieren mayor trascendencia por cuanto se presentan desde la infancia, demostrándose a temprana edad: alteraciones anatómicas, fisiológicas, bioquímicas y conductuales; lo que debiera condicionar, no sólo un cambio en el paradigma de enfrentamiento del RP, sino también en la prevención de la patología cardiovascular (3). 
6 Efecto de Expansión rápida del maxilar en el tratamiento de trastornos respiratorios del sueño en niños, revisión bibliográfica

\section{Anomalías dentomaxilares y creneofaciales}

Los pacientes con TRS presentan con frecuencia problemas deglutorios, alteraciones fonológicas como ceceo, predominio de la respiración bucal, anomalías craneofaciales como: incompetencia labial, cara alargada y angosta, mentón pequeño y triangular, retrognatia mandibular, paladar ojival, compresión maxilar, disminución de la distancia intermolar o canina, vestibuloversión de incisivos superiores, acompañada de retrognatia (16).

Los TRS permiten que se potencie la respiración bucal por sobre la nasal, debido a esto la lengua queda en una posición más baja produciendo un hipodesarrollo de todo el maxilar incluyendo bóveda palatina y cavidad nasal (5,17-19).

Las anormalidades ortodóncicas y craneofaciales comunes entre los niños con TRS no han recibido la atención adecuada por parte de los especialistas $(20,21)$. Las anomalías craneofaciales además de ser un signo clínico, son a su vez un factor predisponente y un factor perpetuante de los TRS pediátricos (5). Esta desarmonía maxilar debería ayudar al clínico a realizar un diagnóstico precoz y derivar para tratamiento de TRS, consciente de que puede mejorar el potencial cognitivo y social a largo plazo y calidad de vida, por lo tanto, cuanto antes un niño se trate, mejor será su desarrollo integral. (10)

\section{Tratamiento de TRS}

El tratamiento de los TRS se basa en disminuir o paliar el aumento de la resistencia de la vía aérea superior. Existen tres formas de tratamiento estudiadas en los TRS: 1) tratamiento medicamentoso, 2) quirúrgico; y 3) ventilación no invasiva (15).

1. El tratamiento farmacológico se basa en el uso de corticoides inhaladores nasales o de montelukast (22).

2. El tratamiento quirúrgico (en general adenotonsilectomía) ha sido recomendado como primera línea, sin embargo, existe cada vez más evidencia que la cirugía no es infalible, en especial en grupos de alto riesgo como los pacientes obesos. Un estudio multicéntrico que incluyó a 578 niños demostró una tasa de éxito post adenotonsilectomía baja, con una alta tasa de prevalencia de obesidad en la muestra (23).

3. El uso de ventilación no invasiva está reservado actualmente para cuadros muy severos, obesidad o pacientes con patologías de base, como por ejemplo los niños con enfermedades neuromusculares (24). 
Por otro lado, el efecto del tratamiento sobre las consecuencias de los TRS puede depender de la precocidad con la cual éste sea iniciado. Es decir, existe la hipótesis que un tratamiento muy tardío no logra revertir del todo los daños causados por los TRS mediante los mecanismos anteriormente señalados (25).

\section{Expansión maxilar como opción terapeutica de los TRS}

La expansión rápida maxilar (ERM) es un procedimiento ortopédico diseñado para aumentar el diámetro transversal del paladar duro por el estímulo de la sutura media palatina, obteniendo como resultado colateral un aumento en el área trasversal de la fosa nasal, también beneficioso en estos casos. Después de 3 a 4 meses postexpansión, una sutura mineralizada normal se consolidará de nuevo. La ERM se utiliza en niños con constricción maxilar y maloclusión dental, es decir, con diámetros trasversales disminuidos o mordidas cruzadas a nivel posterior. Actualmente este procedimiento es una promesa como un tratamiento alternativo de TRS en niños (10). Cuando la ERM se ha aplicado en niños entre 6 y 12 años, con alteraciones esqueletales, independiente de su clase molar, se ha demostrado mejoras significativas de los signos y síntomas de los TRS, así como de los parámetros polisomnográficos $(26,27)$.

Un estudio comparó un grupo sometido a cirugía de amígdalas en niños entre 6 y 12 años, seguida de ERM y otro sometido a un protocolo con la secuencia contraria. Los resultados del examen clínico y polisomnográfíco revelaron mejoras en el segundo grupo, en el que dos pacientes fueron tratados con éxito sin la necesidad de cirugía (28). Es muy importante establecer que con el procedimiento de ERM el volumen total del complejo nasomaxilar se incrementa un $12 \%$, el volumen nasal en un $17 \%$, y el volumen del maxilar en un 10,6 \% (29).

La reducción en los síntomas de los TRS se podría relacionar con el movimiento hacia abajo y hacia adelante de los maxilares causada por la ERM, lo que conduce a un aumento en las cámaras nasales y el flujo de aire mejoraría (20). El efecto ortopédico que produce conduce a un aumento tanto en el espacio nasofaríngeo y un beneficio en la posición de la lengua (30). Esta ampliación del hueso maxilar es generada a través de la osteogénesis por distracción, que se ve favorecida por el hecho de que esta región se compone de hueso compacto lateralmente y tejido fibroso con fibras de colágeno, fibroblastos y vasos sanguíneos en el centro (20).

Debe subrayarse que aproximadamente el $15 \%$ de los pacientes continúan con síntomas respiratorios, como el RP, después de la cirugía de adenotonsilectomía 
8 Efecto de Expansión rápida del maxilar en el tratamiento de trastornos respiratorios del sueño en niños, revisión bibliográfica

o experimentan el regreso de este problema en la edad adulta, sobre todo aquellos pacientes que se sometieron sólo a cirugía y no a ERM (28).

La ERM se obtiene fácilmente hasta los 10 años de edad, no así en edades más tardías (12-18 años) (31,32), esto debido a la anatomía maxilar en pacientes de mayor edad. Existe una alta densidad en la unión de las líneas festoneadas que permite fusión de la sutura media palatina alrededor de los 10 años de edad con variaciones individuales. Por tanto, la oportunidad de tratamiento es clave cuando se trata de pacientes infantiles con TRS, es un momento único que no se puede desaprovechar $(31,32)$.

\section{Conclusiones}

Un factor predisponente en el desarrollo y también en la progresión de los TRS pediátricos es la falta de armonía craneofacial, en niños en desarrollo. Algunas son alteraciones como compresión maxilar, retrognatia, falta de desarrollo nasal, falta de desarrollo sagital del maxilar, entre otras.

La expansión rápida del maxilar se presenta como una alternativa de tratamiento ya que el efecto ortopédico que produce, conduce a un aumento tanto en el espacio nasofaríngeo y un beneficio en la posición de la lengua evitando SAOS residual en pacientes pediátricos.

\section{Referencias}

1. Urquhart D, Tan H. Sleep disordered breathing at the extremes of age: infancy. Breathe. 2016; 12(1):1-11.

2. Brockmann P, Urschitz M, Schlaud M, Poets C. Primary snoring in school children: prevalence and neurocognitive impairments. Sleep Breath. 2012; 16:23-9.

3. Santamaria A, Astudillo D. Roncopatía primaria y sus implicancias clínicas: fin al paradigma de entidad inocua. Rev Otorrinolaringol Cir Cabeza Cuello. 2014; 74:181-90.

4. Liu, C., Kang, W., Zhang, S. et al. Mandibular Advancement Devices Prevent the Adverse Cardiac Effects of Obstructive Sleep Apnea-Hypopnea Syndrome (OSAHS). Sci Rep. 2020; 10:3394. 
5. Katyal V, Pamula Y, Martin AJ, Daynes CN, Kennedy JD, Sampson WJ. Craniofacial and upper airway morphology in pediatric sleep-disordered breathing: Systematic review and meta-analysis. Am J Orthod Dentofacial Orthop. 2013; 143(1):20-30.

6. Prades-Morera E, Esteller E. Clinica de los trastornos respiratorios del sueño en niños. Acta Otorrinolaringol Esp. 2010; 61:22-5.

7. DelRosso L. Epidemiology and Diagnosis of Pediatric Obstructive Sleep Apnea. Current Problems in Pediatric and Adolescent Health Care. 2016; 46(1):2-6.

8. Sang-Youp L, Jeong-Whun K. Nasopharyngeal Width and Its Association with SleepDisordered Breathing Symptoms in Children. Clinical and Experimental Otorhinolaryngology. 2019; 12(4):399-404.

9. Wu Y, Feng G, Xu Z, Li X, Zheng L, Ge W, et al. Identification of different clinical faces of obstructive sleep apnea in children. International Journal of Pediatric Otorhinolaryngology. 2019; 127:109621.

10. Marcus CL, Brooks LJ, Draper KA, Gozal D, Halbower AC, Jones J. Diagnosis and management of childhood obstructive sleep apnea syndrome. Pediatrics. 2012; 130(3):714-55.

11. Lee CH, Kim YJ, Lee SB, Yoo CK, Kim HM. Psychological screening for the children with habitual snoring. Int J Pediatr Otorhinolaryngol. 2014; 78(12):2145-50.

12. Brockmann P, Schlaud M, Poets C, Urschitz M. Predicting poor school performance in children suspected for sleep-disordered breathing. Sleep Med. 2015; 16(9):1077-83.

13. Kun-Tai K, Shuenn-Nan C, Wen-Chin W, Pei-Lin L, Wei-Chung H, Comparisons of Office and 24-Hour Ambulatory Blood Pressure Monitoring in Children with Obstructive Sleep Apnea. The Journal of Pediatrics. 2017; 182:177-83.

14. Tan H, Alonso Alvarez MT, Saoussoglou M, Weber S, Kaditis A. When and why to treat the child who snores? Pediatr Pulmonol. 2017; 52:399-412.

15. Brockmann P. Consecuencias neurocognitivas de los trastornos respiratorios del sueño. Neumol Pediatr. 2012; 7(2):44-7.

16. Kim D, Rhee CS, Yun P, Kim JW. Adenotonsillar hypertrophy as a risk factor of dentofacial abnormality in Korean children. Eur Arch Otorhinolaryngol. 2015; 272:3311-6. 
Efecto de Expansión rápida del maxilar en el tratamiento de trastornos respiratorios del sueño en niños, revisión bibliográfica

17. Uma B, Raghavendra S. Comparison of soft-tissue, dental, and skeletal characteristics in children with and without tongue thrusting habit. Contemp Clin Dent. 2013; 4(1):2-6.

18. Huynh N, Morton PD, Rompre PH, Papadakis A, Remise C. Associations between sleep-disordered breathing symptoms and facial and dental morphometry, assessed with screening examinations. Am J Orthod Dentofacial Orthop. 2011; 140:762-70.

19. Löfstrand-Tideström B, Hultcrantz E. The development of snoring and sleep related breathing distress from 4 to 6 years in a cohort of Swedish children. International Journal of Pediatric Otorhinolaryngology. 2007; 71(7):1025-33.

20. Giannasi LC, Santos IR, Alfaya TA, Bussadori SK, Leitão-Filho FS, de Oliveira LV. Effect of a rapid maxillary expansion onsnoring and sleep in children: a pilot study. Cranio. 2015;33(3):169-73.

21. Graf I, Schumann U, Neuschulz J, Höfer K, Ritter L, Braumann B. Sleep-disordered breathing in orthodontic practice: Prevalence of snoring in children and morphological findings. $J$ Orofac Orthop. 2016; 77(2):129-37.

22. Kuhle S, Urschitz MS, Eitner S, Poets CF. Interventions for obstructive sleep apnea in children: A systematic review. Sleep Med Rev. 2009; 13:123-31.

23. Bhattacharjee R, Kheirandish-Gozal L, Spruyt K, Mitchell RB, Promchiarak J, Simakajornboon $\mathrm{N}$, et al. Adenotonsillectomy outcomes in treatment of obstructive sleep apnea in children: a multicenter retrospective study. Am J Respir Crit Care Med. 2010; 182:676-83.

24. Brockmann P, Salinas P, Holmgren N, Prado F. Utilidad de la clínica para el diagnóstico de trastornos respiratorios del sueño en niños con enfermedades neuromusculares. Rev Chil Pediatr. 2011; 4:319-28.

25. Beebe DW, Ris MD, Kramer ME, Long E, Amin R. The association between sleep disordered breathing, academic arades, and cognitive and behavioral functioning among overweight subjects during middle to late childhood. Sleep. 2010; 33(11):1447-56.

26. Sánchez-Súcar AM, Sánchez-Súcar FB, Almerich-Silla JM, et al. Effect of rapid maxillary expansion on sleep apnea-hypopnea syndrome in growing patients. A meta-analysis. J Clin Exp Dent. 2019; 11(8):e759-e67.

27. Villa MP, Malagola C, Pagani J, et al. Rapid maxillary expansion in children with obstructive sleep apnea syndrome: 12-month follow-up. Sleep Med. 2007; 8(2):128-34. 
28. Guilleminault C, Huang YS, Quo S, Monteyrol PJ, Lin CH. Teenage sleep-disordered breathing: recurrence of syndrome. Sleep Med. 2013; 14(1):37-44.

29. Bouserhal J, Bassil-Nassif N, Tauk A, Will L, Limm M. Three-dimensional changes of the naso-maxillary complex following rapid maxillary expansion. Angle Orthodontist. 2014; 84(1):88-95.

30. Alrejaye N, Gao J, Hatcher D, Oberoi S. Effect of maxillary expansion and protraction on the oropharyngeal airway in individuals with non-syndromic cleft palate with or without cleft lip. PLoS One. 2019; 14(7):e0213328.

31. Angelieri F, Cevidanes L, Franchi L, Gonçalves J, Benavides E, McNamara J. Midpalatal suture maturation: Classification method for individual assessment before rapid maxillary expansion. Am J Orthod Dentofacial Orthop. 2013; 144(5):759-69.

32. Guilleminault C, Monteyrol PJ, Huynh NT, Pirelli P, Quo S, Li K. Adeno-tonsillectomy and rapid maxillary distraction in pre-pubertal children, a pilot study. Sleep Breath. 2011; 15(2):173-7. 